\title{
Direct Computation of Shape from Shading
}

\author{
Michael J. Brooks and Wojciech Chojnacki \\ \{mjb,wojtek\}@cs.adelaide.edu.au \\ Department of Computer Science \\ University of Adelaide, SA 5005, Australia
}

\begin{abstract}
A direct method for the recovery of surface shape from image shading is presented. The method in its basic form is applicable to shading patterns obtained when a distant overhead point light-source illuminates a convex or concave Lambertian surface possessing a single extremal point at which surface height has either a local maximum or local minimum. A key quality of the method is its ability to operate without explicit prerequisite information concerning the shape of the sought-after surface. Consideration is also given to the manipulation of images of more complex surface shapes.
\end{abstract}

\section{Introduction}

\subsection{Motivation}

A major challenge in computer vision is to develop a versatile shape from shading algorithm able to operate effectively in the absence of substantial prerequisite information [8]. Such an algorithm has so far proven elusive. Foundational mathematical results on the initial information needed to solve the shape from shading problem appear to be at odds with the apparent human capacity to operate with less than minimal prerequisite information. While humans are not always successfully able to interpret shading patterns, they are frequently able to perceive surface shape reasonably accurately from image shading. This is often achieved without prior knowledge of lighting conditions, surface lustre, etc. Reconciliation is perhaps to be found in the speculation that humans make certain assumptions that prove fairly robust in determining shape from shading.

A most serious concern is that nearly all shape-fromshading methods need substantial prior knowledge of surface shape-the very information being sought. Thus, methods based on conventional regularisation techniques typically need to be given some surface normals of the sought-after shape. (Although the known presence of an occluding boundary enables such normals to be computed around the periphery of the surface image.) Local methods, such as [11], are unusual in not needing prerequisite shape information. However, they have failed to prove effective.
To gain further insight into prerequisite requirements and the shape-from-shading problem, it is instructive to examine the approaches of Horn [7] and Bruckstein [3].

The foundational method of Horn requires that surface normals be given along some initial curve in the image. Other normals may subsequently be computed along paths sprouting from the initial curve. These paths called base characteristic curves are, in the case of the Lambertian reflectance map, projections onto the image domain of the solution surface's lines of steepest ascent with respect to the illuminant direction. The method of Bruckstein requires specification of a loop in the image domain around which surface height is constant. The loop might, for example, surround the point of maximal brightness. Successive contours of constant height could then be computed by expanding outward (or contracting inward) from the initial loop.

The rather onerous initial requirements of these fundamental algorithms are formally necessary if they are to have fairly general application. There are, however, situations in which the shape from shading problem is well posed in the absence of the initial information on shape required by these algorithms. Foundational results on uniqueness reveal that under certain conditions shape may be determined from shading without the prior knowledge of explicit shape information, such as specific height values or surface normals (see [4], [9]). (Note, however, that an implicit assumption is always necessary as to the form of admissible surfaces.) By way of example, suppose that a given image is known to have been formed by illuminating a convex Lambertian surface with a distant overhead point source. If the image happened to be that of a wholly visible hemisphere, exhibiting a single point of maximal image brightness, the method of Horn would require an initial loop of known normals, surrounding the image point of maximal brightness, if it were to recover the shape of the hemisphere. Similarly, the method of Bruckstein would need to be given a loop in the image domain, again surrounding the image point of maximal brightness, around which surface height was known to be constant. Importantly, however, neither of these shape clues is in this situation necessary: the shape is computable 
without explicit initial shape data [2].

Our motivation in this ongoing work is to find shape from shading algorithms, perhaps operating in a restricted environment, that require no explicit initial shape information. We propose a method of shape recovery that can be viewed as a direct variational method, in contrast with the many existing, and fundamentally different, non-direct variational methods. It should be noted that aspects of [5], [6], and [10] relate closely to our work, although the approach taken here is quite different.

\subsection{Prerequisites}

The shape-from-shading problem may be expressed mathematically in terms of a first-order partial differential equation. Specifically, a function $u(x, y)$ is sought, representing surface depth in the direction of the $z$-axis, that satisfies the image irradiance equation $R\left(u_{x}, u_{y}\right)=E(x, y)$ over $\Omega$. Here $E$ is an image formed by (orthographic) projection of light onto a plane parallel to the $x y$-plane, situated above the surface, $R$ is the reflectance map relating image brightness to surface orientation, and $\Omega$ is the image domain. In this formulation, it is implicitly assumed that light sources are infinitely far away, and internal surface reflections are disallowed.

Assume that a distant overhead point-source illuminates a Lambertian surface of unit albedo. Then the corresponding image irradiance equation is

$$
\left(u_{x}^{2}+u_{y}^{2}+1\right)^{-1 / 2}=E(x, y) .
$$

Noting that $0<E(x, y) \leq 1$, we may safely let

$$
\mathcal{E}(x, y)=E^{-2}(x, y)-1
$$

and express (1) as the eikonal equation

$$
u_{x}^{2}+u_{y}^{2}=\mathcal{E}(x, y)
$$

Any $C^{2}$ function satisfying (3) over $\Omega$ will be referred to as a solution. For a given function $u$, if $X \in \Omega$ is such that $u_{x}(X)=u_{y}(X)=0$, then $X$ will be called a critical point of $u$. It is readily seen from equations (2) and (3) that any point at which $E$ equals 1 (that is, at which image brightness is maximal) is critical for every solution. Conventionally, a point in an image at which $E$ attains 1 is called singular. Later, given a function $u$, we will be particularly interested in non-degenerate, or hyperbolic critical points $X \in \Omega$ for which $\Delta(X)=u_{x x}(X) u_{y y}(X)-u_{x y}^{2}(X) \neq 0$. From the standpoint of differential topology, functions all of whose critical points are non-degenerate are typical. If $\Delta(X)>0$, then always $u_{x x}(X)+u_{y y}(X) \neq 0$. A critical point $X$ for which $\Delta(X)>0$ and $u_{x x}(X)+u_{y y}(X)<0$ will be called convex, and a critical point $X$ such that $\Delta(X)>0$ and $u_{x x}(X)+u_{y y}(X)>0$ will be termed concave. A critical point $X$ with $\Delta(X)<0$ will be called saddle. When $X$ is a critical point of $u$, then depending on whether $u(X)$ is convex, concave, or saddle, $u$ will at $X$ exhibit a local maximum, local minimum, or saddle respectively.

Note that if $u$ is a solution to (3), then so too are functions of the form $\pm u+k$, where $k$ is a constant. Of course, different values of the constant $k$ in the above expression leave the shape unchanged, so we may regard $-u$ as being the only one of these surfaces different in shape to $u$, and this we shall call the dual solution.

\section{An integration scheme for height recovery}

In this section, we present a method that recovers surface height by sweeping outwards from a non-saddle singular point in the image. Note that while Horn's method is unable to integrate outward from a singular point, the approach adopted here is nevertheless intimately related to the method of characteristic strips.

The essence of the method is as follows. Suppose that two points in the domain of the image are linked via a base characteristic curve that is wholly contained in the domain. An expression is derived whose integral along the base characteristic curve connecting these points equals the absolute value of the difference of the surface heights at the points in question. Moreover, the expression is such that its integral along any other curve in the domain connecting the two points is not smaller than this value. Thus the relative height of the two points can be determined by finding the minimum of integrals taken over all paths in the domain connecting them.

Given that the pattern of base characteristics is unknown a priori, the challenge is thus to devise an implementation, utilising the method outlined above, that efficiently searches through all possible paths in the discrete domain seeking the one(s) with maximum integral. Below we present mathematical fundamentals and an associated computational scheme.

\subsection{Underlying mathematics}

The method is based on the following crucial result:

Theorem 1 If a function $u$ satisfies (3) in a domain $\Omega$, and $X$ and $Y$ are points in $\Omega$ that can be joined by $a$ base characteristic curve wholly contained in $\Omega$, then the relative depth $|u(X)-u(Y)|$ between points on the graph of $u$, having $X$ and $Y$ as images, is given by

$$
|u(X)-u(Y)|=\min _{\gamma} \int_{\gamma} \sqrt{\mathcal{E}} d l,
$$

where the minimum is taken over all piecewise smooth curves $\gamma$ in $\Omega$ joining $X$ and $Y$, and the integration is taken with respect to the standard line measure. 
For the proof we refer the reader to [1].

A surface having an isolated maximum (minimum) and being such that every point in the surface's image can be joined by a base characteristic curve with the singular point at which surface height is maximal (minimal) will be called convex (concave). The above definition is not intrinsically geometric as it depends on the direction from which the surface is illuminated. Note that, according to this definition, the surface shown in Figure 1a is convex.

Let $\Omega$ be an open domain in the $x y$-plane, and $\mathcal{E}$ be a non-negative function on $\Omega$. For every pair of points $X, Y \in \Omega$, put

$$
d(X, Y)=\min _{\gamma} \int_{\gamma} \sqrt{\mathcal{E}} d l,
$$

where the minimum is taken over all piecewise smooth curves $\gamma$ in $\Omega$ joining $X$ and $Y$. The function $d$ thus defined is a metric in $\Omega$. It can easily be inferred from Theorem 1 that if a function $u$ on $\Omega$ satisfies equation (3) and has a single critical point $S$, then, for every point $X \in \Omega$,

$$
u(X)=u(S)-d(X, S)
$$

whenever the graph of $u$ is convex, and

$$
u(X)=u(S)+d(X, S)
$$

whenever the graph of $u$ is concave.

\subsection{Computational scheme}

We now present a computational scheme based on the above theorem. The scheme involves a local cooperative computation in which an estimate of height at some point in the domain is obtained by successive consideration of image values and height estimates in a small neighbourhood of the point. It is assumed that a base characteristic curve connects the singular point to the point under consideration.

Let $S$ denote the singular point. Given $X \in \Omega$, let $\mathcal{N}(X)$ denote the neighbourhood of $X$ comprising the eight nearest neighbours of $X$ in a rectangular grid and $X$ itself. For $X, Y \in \Omega$, let $I_{X, Y}$ be the integral of $\sqrt{\mathcal{E}}$ over the interval joining $X$ with $Y$. Given a non-negative integer $n$ and $X \in \Omega$, let $U_{X}^{(n)}$ denote the $n$th estimate of height at $X$. The height estimates $U_{X}^{(n)}$ are computed recursively as follows:

$$
U_{X}^{(0)}= \begin{cases}0 & \text { if } X=S, \\ -\infty & \text { if } X \in \Omega \backslash\{S\}\end{cases}
$$

and, for each $n$,

$$
U_{X}^{(n+1)}=\max _{Y \in \mathcal{N}(X)}\left\{U_{Y}^{(n)}-I_{X, Y}\right\}
$$

The recursion terminates when, for some $n, U_{X}^{(n)}=U_{X}^{(n+1)}$ for all $X \in \Omega$. The above scheme implements (6) using dynamical programming principles of efficient computation.

At the local level, the above computation proceeds as follows. Given $X \in \Omega$, suppose that each neighbour $Y$ of $X$ in the $3 \times 3$ window $\mathcal{N}(X)$ has been assigned a height value. Associated with $Y$ is a new candidate for the height at $X$ obtained by subtracting the integral $I_{X, Y}$ from the current height estimate at $Y$. A new height value for $X$ is then obtained by choosing the maximum of these candidate height values. Early on in the computation, height values are computed by sweeping out from a singular point, which has arbitrarily been assigned initial height value zero. Heights across the image are recomputed as many times as is necessary to reach a steady state. In this way, complex and twisting paths are eventually explored if the base characteristics exhibit such a form. Note that the scheme is amenable to parallelism as it takes a Jacobian form whereby recent updates are not immediately utilised within a given iteration.

\section{Application of the integration scheme}

The question now arises as to how the above integration scheme might be applied to an image for which there is known to exist a smooth solution satisfying equation (3). This simple question raises many issues.

In this paper, we shall confine our attention to those smooth surfaces that have base characteristic curves pointing inward at the periphery of the domain. We may regard such surfaces as having a convex skirt around their boundary [9]. Such a skirt may or may not extend far enough to become an occluding boundary. Denote by $N_{+}, N_{-}$, and $N_{ \pm}$the number of a given surface's critical points that are convex, concave, and saddle, respectively, and by $N_{S}$ the total number of critical points. If the surface has a convex skirt and is defined over a simply connected domain, then

$$
N_{+}+N_{-}-N_{ \pm}=1
$$

(see [3]). This together with the obvious equality $N_{S}=$ $N_{+}+N_{-}+N_{ \pm}$implies that

$$
N_{S}=2 N_{ \pm}+1 .
$$

We thus see that smooth surfaces having a convex skirt must always have an odd number of critical points. The same result applies to the duals possessing a concave skirt.

We may now characterise the combinations of critical points exhibited by smooth shapes having a convex skirt. Starting with surfaces exhibiting only one convex critical point, with which we associate the set $\{+\}$, we may generate new surfaces by successively adding - at the symbolic level一arbitrarily many of either $\{+ \pm\}$ or $\{- \pm\}$. Thus 
the set $\{++- \pm \pm\}$ is legitimate, signifying five critical points-two convex, two saddle, and one concave. In general, several shapes of somewhat different character will share the same symbolic description. The ordering of the signs being irrelevant, we adopt here a convention according to which + signs go first, followed by - signs, and \pm signs. With this convention, successive sets are as follows:

$$
\begin{aligned}
& \{+\} \\
& \{++ \pm\},\{+- \pm\} \\
& \{+++ \pm \pm\},\{++- \pm \pm\},\{+-- \pm \pm\}
\end{aligned}
$$

If an image possesses no singular point, then the integration method clearly cannot be used. By way of example, we now consider the applicability of the previously specified method to images possessing either one or three singular points.

\subsection{Images possessing one singular point}

In the event that the image exhibits a single point of maximal brightness, we may utilise the integration scheme to obtain a surface. There is, however, no guarantee that the resulting surface constitutes a solution. For example, application of the integration technique will lead to an incorrect result when the given image is generated by a surface with a saddle critical point - the solution surface will then be either convex or concave, and as such will be fundamentally different from the initial surface.

It can be inferred from a result of [2] that any image can locally be modified in such a way that the resulting shading pattern is impossible in that it is not an image of any shape; moreover, if the initial image contains a singular point, then the modification can be made in such a fashion that the singular point will remain intact. When the integration method is applied to an impossible image with a singular point, then the resulting surface will not be smooth and/or will not satisfy the image irradiance equation.

It is therefore clear that the developed integration technique provides a means of finding a convex solution if such a solution exists. The solution is determined in that neighbourhood of a non-saddle critical point that is covered by the base characteristics linked to that point. The dual concave solution is then readily obtained.

Figure 1 displays the integration method at work. When applied to a synthetic image, obtained via (3), of the convex surface depicted in Figure 1a, the procedure yields the surface shown in Figure 1b. As we can see, the estimate approximates well the original shape.

\subsection{Images possessing three singular points}

In the event that the image exhibits three points of maximal brightness, any solution having a convex skirt is either

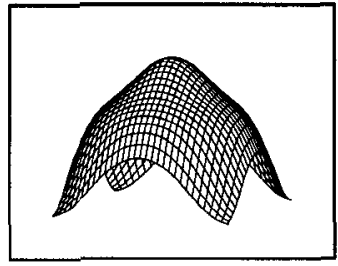

a

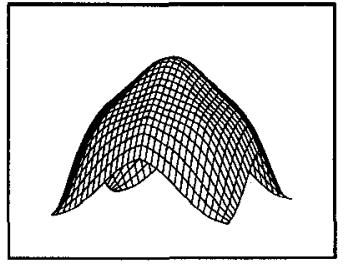

b
Figure 1. (a) depicts a convex surface, while (b) depicts the result of an application of the integration procedure to its image.

of type $\{++ \pm\}$ or of type $\{+- \pm\}$. The question then arises as to how such a solution may be derived given that the type of each singular point is unknown.

Suppose we integrate across the whole domain from one of the singular points, thereby generating a surface. When the starting singular point is not saddle, such a surface will in general constitute a solution only over a portion of the domain. For this reason, the surface will be termed a partial solution. The singular point from which it was generated will be dubbed the source. Later, this terminology will also be applied to saddle singular points and corresponding surfaces.

In the event that the source point is not saddle, the partial solution will be an actual solution in a region covered by base characteristics linked to the source. The surface computed outside this region will in general fail to constitute a solution. In the event that the source point is saddle, the partial solution will fail to match a solution nearly everywhere. Correct height values will be computed only along the two base characteristics that link the saddle point with the other (non-saddle) singular points.

A typical situation that arises is depicted in Figure 2. Figure 2a displays a shape with a convex skirt, whose image has three singular points. By taking successively each of these points as source, partial solutions can be developed, as shown in Figures $2 \mathrm{~b}, 2 \mathrm{c}$, and $2 \mathrm{~d}$. None of these partial solutions constitutes a solution over the whole domain. We see, however, that surface height is correctly determined in an ample neighbourhood of each convex source. This suggests that an actual solution should be represented as a combination of the partial solutions with a convex source. As we shall soon see, such a representation can indeed be derived. But first, we identify the non-saddle points, or equivalently, determine which of the three singular points is saddle. Before formulating a theorem that will address this issue, we introduce some notation.

Let $\Omega$ be a simply connected domain, and $\mathcal{E}$ be a nonnegative function on $\Omega$, and $d$ be the metric given by (5). If $S \in \Omega$ is a source in our terminology, then the correspond- 


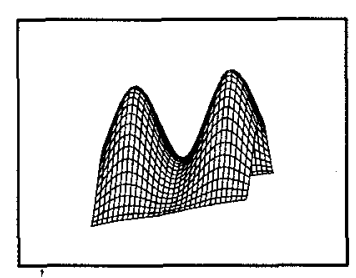

a

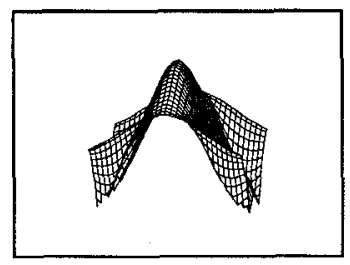

C

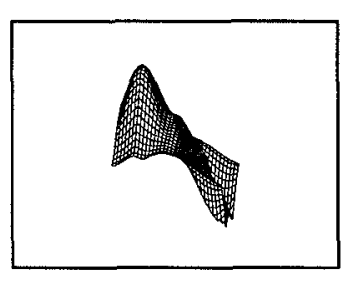

b

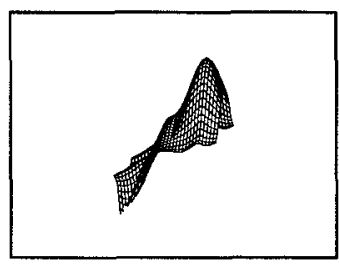

d
Figure 2. (a) depicts a surface exhibiting two peaks; given an image of (a) formed via (3), figures (b), (c) and (d) display the partial solutions obtained by treating successively each singular point as source.

ing partial solution $U_{S}$ takes the form

$$
U_{S}(X)=d(S, X) \quad(X \in \Omega) .
$$

For any three points $X_{i}(i=1,2,3)$ in $\Omega$ and any $i=$ $1,2,3$, put

$$
D\left(X_{i}\right)=d\left(X_{i}, X_{j}\right)+d\left(X_{i}, X_{k}\right),
$$

where $j, k \in\{1,2,3\} \backslash\{i\}$.

Theorem 2 If a function $u$ satisfies (3) in $\Omega$, has a convex skirt, and has three different critical points $X_{i}(i=1,2,3)$, then the point $X_{i}$ for which $D\left(X_{i}\right)$ is minimal is saddle.

With Theorem 2 at hand, it is now easy to determine which of the singular points is saddle. In the next step, we find out whether one of the remaining points is concave, and if so, immediately derive a solution shape. Crucial here is the observation that for a $\{+- \pm\}$ surface all points in the image domain can be joined to the convex singular point by base characteristic curves. Thus, if the initial surface is of type $\{+- \pm\}$, then the partial solution with a convex source is a genuine solution, and exhibits a concavity around the concave singular point. Inspection reveals that such a concavity cannot be exhibited in the other partial solution with a non-saddle source in the $\{+- \pm\}$ case, or in the two partial solutions with a non-saddle source in the $\{++ \pm\}$ case. We thus see that the initial shape is of type $\{+- \pm\}$ if and only if one of the partial solutions with a non-saddle source exhibits a concavity, in which case that partial solution is a genuine solution.

In the event that there is no concave singular point, a solution, necessarily of type $\{++ \pm\}$, may be derived from two partial solutions with a convex source as follows. Offset the height of each partial solution so that both the solutions have equal height at the saddle point, and then, for each image point, select the height value from the partial surface having maximum height. The resulting surface is a desired solution. The validity of this procedure is guaranteed by the following theorem:

Theorem 3 If a function u satisfies (3) in $\Omega$, has a convex skirt, and is of type $\{++ \pm\}$ with two convex critical points $X_{1}$ and $X_{2}$, and with a saddle critical point $X_{3}$, then

$$
\begin{aligned}
u(X)= & \max \left\{U_{X_{1}}\left(X_{3}\right)-U_{X_{1}}(X),\right. \\
& \left.U_{X_{2}}\left(X_{3}\right)-U_{X_{2}}(X)\right\}+u\left(X_{3}\right) .
\end{aligned}
$$

The procedure for interpreting images having three singular points of known location, but of unknown type, may now be summarised as follows:

- Locate the positions in the image of the three singular points.

- Derive three partial solutions, extending across the domain of the image, by successively treating each of the three singular points as source.

- Compute, for each partial solution, the neighbourhood sum, this being defined as the sum of the absolute height differences between the source and the other singular points.

- Locate the saddle point as the source whose partial solution generates the smallest neighbourhood sum.

- Discard the partial solution with saddle source.

- For each of the remaining (two) partial solutions, inspect the generated shape in a neighbourhood of the singular point which is neither source nor saddle. If the shape is essentially concave, then the singular point is indeed concave. Furthermore, the partial solution with the corresponding convex source is a genuine solution of type $\{+- \pm\}$.

- In the event that there is no concave singular point, offset in height each of the partial solutions with convex source so that both solutions have equal height at the saddle point, thereby registering two new partial solutions. Derive a function that is the maximum of the two newly obtained partial solutions. This will be a solution of type $\{++ \pm\}$. 


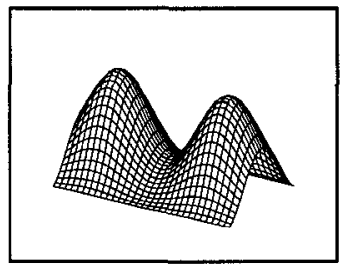

a

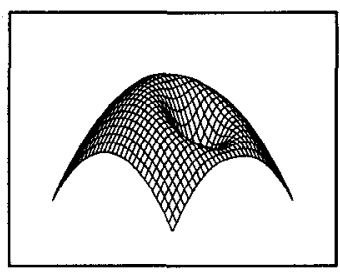

C

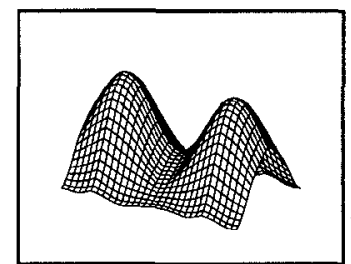

b

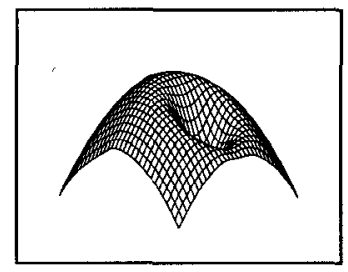

d
Figure 3. (a) depicts a surface exhibiting two peaks, while (b) displays the estimate obtained from an image of (a); likewise (d) shows the surface derived using an image of (c).

- Check whether the final estimated surface is a solution to the image irradiance equation. If so, note that its dual is also a solution.

Figure 3 a shows an example of a $\{++ \pm\}$ surface, while Figure $3 \mathrm{~b}$ shows the result obtained by applying the above procedure to its image. Similarly, the central portion of Figure $3 c$ depicts a surface of type $\{+- \pm\}$, with a corresponding estimate given in Figure 3d. Surfaces recovered reveal relatively little error, and suggest that the method holds promise.

We conclude by remarking that the method presented in this paper may be generalised in a straightforward manner to cover the case of an arbitrary (odd) number of singular points in the image domain. The analysis of the case of three singular points and the techniques developed herein prove sufficient to deal with the general case. Subsequent work will deal with non-overhead lighting.

\section{Acknowledgements}

Part of this research was conducted while the first-named author was on sabbatical leave at INRIA, Sophia Antipolis, France. This author is indebted to Prof. Olivier Faugeras for hosting the visit, and to Mads Nielsen for valuable discussions.

\section{References}

[1] M. J. Brooks and W. Chojnacki, Direct computation from shape from shading, Tech. Report 2176, Institut
National de Recherche en Informatique and en $\mathrm{Au}$ tomatique, Sophia Antipolis, France, January 1994.

[2] M. J. Brooks, W. Chojnacki, and R. Kozera, Impossible and ambiguous shading patterns, International Journal of Computer Vision 7 (1992), no. 2, 119-126.

[3] A. M. Bruckstein, On shape from shading, Computer Vision, Graphics and Image Processing 44 (1988), no. $2,139-154$.

[4] A. R. Bruss, The eikonal equation: some results applicable to computer vision, Journal of Mathematical Physics 23 (1982), no. 5, 890-896.

[5] P. Dupuis and J. Oliensis, Direct method for reconstructing shape from shading, Proceedings of the DARPA Image Understanding Workshop (San Diego, CA, January 26-29, 1992), Morgan Kaufman, San Mateo, CA, 1992, pp. 563-571.

[6] _ Direct method for reconstructing shape from shading, Proceedings of the IEEE Conference on Computer Vision and Pattern Recognition (Champaign, IL, June 15-18, 1992), IEEE Computer Society Press, Los Alamitos, CA, 1992, pp. 453-458.

[7] B. K. P. Horn, Obtaining shape from shading information, The Psychology of Computer Vision (P. H. Winston, ed.), McGraw-Hill, New York, 1975, pp. 115155.

[8] B. K. P. Horn and M. J. Brooks (eds.), Shape from shading, MIT Press, Cambridge, MA, 1989, MA.

[9] J. Oliensis, Uniqueness in shape from shading, International Journal of Computer Vision 6 (1991), no. 2, 75-104.

[10] J. Oliensis and P. Dupuis, A global algorithm for shape from shading, Proceedings of the Fourth International Conference on Computer Vision (Berlin, Germany, May 11-14, 1993), IEEE Computer Society Press, Los Alamitos, CA, 1993, pp. 692-701.

[11] A. P. Pentland, Local shading analysis, IEEE Transactions on Pattern Analysis and Machine Intelligence 6 (1984), no. 2, 170-187. 